\title{
Size-dependent stability of iron oxide evaluated through in-situ heating experiments
}

\author{
Cecile Bonifacio Fittz ${ }^{1 *}$, Hasti Majidi ${ }^{1 \#}$ and Klaus van Benthem ${ }^{1}$ \\ 1. University of California Davis, Department of Materials Science And Engineering, Davis, CA 95616, \\ USA \\ *. now at: E.A. Fischione Instruments, Inc. Export, PA 15632, USA \\ \#. now at: Alta Devices, Sunnyvale, CA 94085, USA
}

The properties of nanoscale materials can be significantly different from that of their bulk counterparts, which is attributed to increasing surface to volume ratios for continuously decreasing dimensions, and potentially culminating in quantum confinement [1]. For instance, Navrotsky and co-workers suggest considerable errors in oxygen fugacity of $100-200 \mathrm{~K}$ when using thermodynamic data for bulk phases to calculate reduction-oxidation phase equilibria for various metal oxides at the nanoscale [2].

Iron oxide is ubiquitous in nature and is commonly found in multiple different polymorphs. Potential applications of iron oxides include fuel cells, electrode materials in batteries, and nanoscale magnets. In rocks, $\mathrm{FeO}$ acts as a redox buffer coexisting with $\mathrm{Fe}_{2} \mathrm{O}_{3}$ to form magnetite, i.e., $\mathrm{FeO} \bullet \mathrm{Fe}_{2} \mathrm{O}_{3}$ or $\mathrm{Fe}_{3} \mathrm{O}_{4}$. Bulk $\mathrm{FeO}$ is stable above $560^{\circ} \mathrm{C}$ while it remains metastable at lower temperatures in the multiphase configuration. Navrotsky et al. [2] have employed calorimetric techniques to estimate surface energies for various iron oxides as a function of initial particle size. Aided by thermodynamic data for bulk oxides they indirectly concluded that $\mathrm{FeO}$ is thermodynamically unstable at or below $100 \mathrm{~nm}$.

In this study we have gradually reduced individual $\gamma-\mathrm{Fe}_{2} \mathrm{O}_{3}$ nanoparticles with diameters of roughly 40$50 \mathrm{~nm}$ by in-situ heating experiments in the transmission electron microscope. For comparison, 1dimensional nanochains of $\gamma-\mathrm{Fe}_{2} \mathrm{O}_{3}$ nanoparticles with various lengths were synthesized with a selfsustaining diffusion flame inside a homogeneous magnetic field (see Figure 1a) [3]. Individual nanochains were caught on Protochips MEMS devices [4] for subsequent in-situ heating experiments (Figure $1 \mathrm{~b}$ and $\mathrm{c}$ ). In-situ heating experiments were carried out with an aberration corrected Jeol JEM 2100F STEM instrument, while electron energy-loss spectra of the $\mathrm{Fe}_{2,3}$ and $\mathrm{O} \mathrm{K}$ absorption edges were recorded with a Gatan Tridiem spectrometer. To evaluate the oxidation state for Fe during the continuous reduction experiments, the $\mathrm{L}_{3} / \mathrm{L}_{2}$ white line intensity ratios for the $\mathrm{Fe} \mathrm{L}_{2,3}$ edges were obtained for different temperatures, and subsequently compared to data from the literature obtained using identical analysis routines [6].

The experimental results recorded from chains of particles with varying lengths demonstrate that $\mathrm{FeO}$ is in equilibrium with $\mathrm{Fe}_{3} \mathrm{O}_{4}$ and metallic $\mathrm{Fe}$ between $400^{\circ} \mathrm{C}$ to $800^{\circ} \mathrm{C}$. For individual particles, however, $\gamma-\mathrm{Fe}_{2} \mathrm{O}_{3}$ gradually reduces to $\mathrm{Fe}_{3} \mathrm{O}_{4}$ before it transforms directly to metallic $\mathrm{Fe}$ [5]. In conclusion, the insitu TEM studies reported here provide direct experimental evidence that indeed $\mathrm{FeO}$ is thermodynamically unstable at the nanoscale. However, the $\mathrm{FeO}$ phase can be stabilized by arranging nanoparticles in a 1-dimensional nanochain with a minimal critical length.

The authors acknowledge funding through an NSF Faculty Early CAREER award (DMR-0955638) and a grant from the Army Research Office (\#W911NF1210491; program manager: Dr. David Stepp). Drs. Gautom Das and Ian Kennedy (UC Davis) are gratefully acknowledged for providing the iron oxide nanoparticles and nanochains. Invaluable discussions with Alexandra Navrotsky are appreciated. 
References:

[1] Roduner, E. Chemical Society Reviews, 35 (2006), p. 583.

[2] Navrotsky, A. et al., Science 330 (2010), p. 199.

[3] Das, G.K. et al., Journal of Materials Chemistry A 2 (2014), p. 12974.

[4] Asoro, M.A. et al., ACS Nano 7 (2013), p. 7844.

[5] Bonifacio, C.S. et al., submitted for publication (2017).

[6] Hihath S. et al., Journal of Applied Physics 116 (2014), 084306
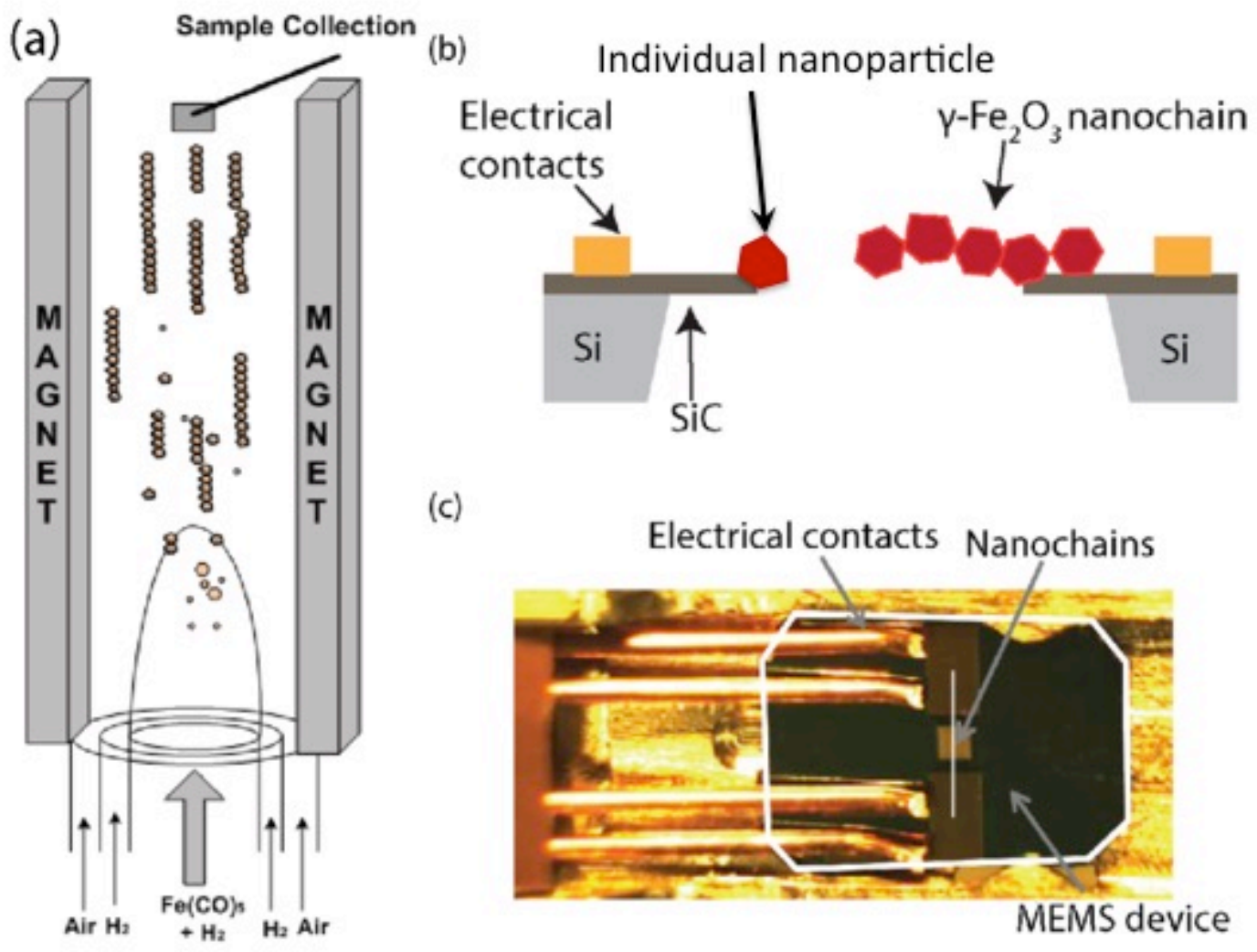

(c)

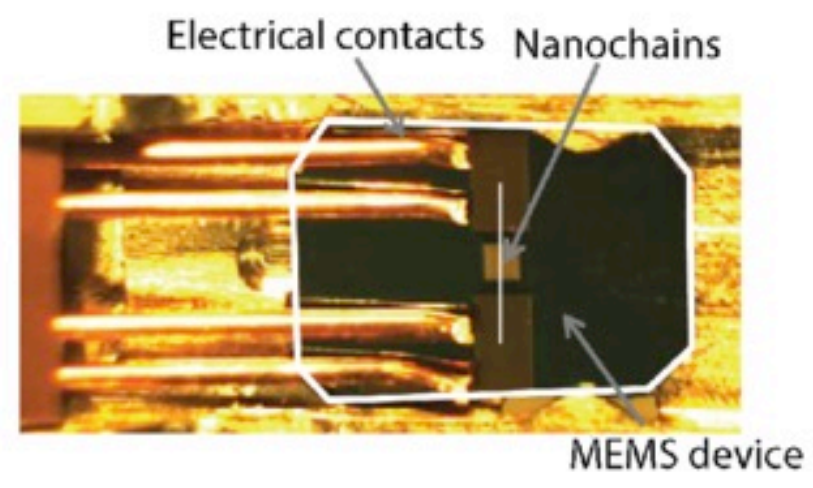

Figure 1. (a) shows the diffusion flame utilized for the synthesis of the 1-dimensional nanochains. (b) is a sketch of the Protochips MEMS device (see picture in panel c) onto which either nanochains or individual nanoparticles were deposited. 